\title{
Correction to: Characterization of three soybean landraces resistant to Asian soybean rust disease
}

\author{
Luciano Nobuhiro Aoyagi • Yukie Muraki • \\ Naoki Yamanaka (1)
}

Published online: 1 August 2020

(C) Springer Nature B.V. 2020

\section{Correction to: Mol Breeding (2020) 40: 53 https://doi.org/10.1007/s11032-020- \\ 01132-w}

The original article unfortunately contains mistakes. In the original Table 1, some values were not correct because of miscalculation. The correct values are presented in new Table 1. Thus, the values in the text were also replaced, 5th page, second column, line 26: '-0.81' and ' -1.25 ' were changed into ' -0.93 ' and ' -1.42 ', respectively; on the same page and column, line 27: ' 1.15 ' and ' 1.16 ' were changed into ' 0.86 ' and ' 0.87 ', respectively; line 36 : ' 0.72 ' and ' 0.73 ' were changed into ' 0.73 ' and ' 0,74 ', respectively; line 47 : ' -0.92 ' and '1.26 ' were changed into ' -1.09 ' and ' -1.47 ', respectively; line 49: ' 1.09 ' and ' 1.07 ' were changed into ' 0.80 ' for both; 6th page, second column, line 4: '-1.17' and '1.30 ' were changed into ' -1.18 ' and ' -1.31 ', respectively; 6th page, second column, line 5: '0.28' was changed into ' 0.29 '; 7th page, first column, line 2: ' 0.67 ' and ' 0.65 ' were changed into ' 0.68 ' and ' 0.66 ', respectively. In the 3rd page, second column, lines 38 and 43, we changed mis-citation of 'Yamanaka et al. (2010)' to correct 'Yamanaka et al. (2019)'. In the 4th page, first column, the line 39 we changed the wrong name 'MRP-
16 ' to the correct 'MRP-13.18'. In the last line wrong citation, 'Supplementary Sheet 1' was changed to 'Table 2'. In the second column, line 9 we changed 'Supplementary Sheet 2' to 'Table 3'. In the 5th page, second column, line 37 there was correction of the citation, 'Table 2' to 'Table 1', and in the 10th page, first column, line 27 , the name of the genotype was wrong and replaced by the correct name 'PI 416764'. According to this change, the 10th page, second column, line 4: 'These facts, along with' was changed to 'However,'. Unnecessary 'WC61'in the 10th page, first column, line 29 was excluded.

In the references, we excluded the wrong reference 'Yamanaka N (2015), and in the 2nd reference we excluded an unnecessary author. The addition of the second author's name 'Kato M,' in the reference 'Yamanaka N, Akamatsu H, Yamaoka Y (2019) JIRCAS website', were made. In the reference, Yamanaka et al. (2010), we inserted a missing part in the title and correct it into 'Development of classification criteria for resistance to soybean rust and differences in virulence among Japanese and Brazilian rust populations.'

The authors of this publication apologize for any inconvenience caused by these mistakes.

The online version of the original article can be found at https://doi.org/10.1007/s11032-020-01132-w

L. N. Aoyagi · Y. Muraki · N. Yamanaka $(\bowtie)$ Japan International Research Center for Agricultural Sciences (JIRCAS), 1-1 Ohwashi, Tsukuba, Ibaraki 305-8686, Japan e-mail: naokiy@affrc.go.jp 
Table 1 Association between Asian soybean rust (ASR) resistance and simple sequence repeat (SSR) markers in BRS $184 \times$ WC2, BRS $184 \times$ WC51, and BRS $184 \times$ WC61 $_{2}$ populations, calculated by one-way ANOVA and regression analysis

\begin{tabular}{|c|c|c|c|c|c|c|c|c|c|c|}
\hline \multirow[t]{2}{*}{ Population } & \multirow{2}{*}{$\begin{array}{l}\text { Resistance } \\
\text { characters* }\end{array}$} & \multirow[t]{2}{*}{ Markers $^{\mathbf{a}}$} & \multirow[t]{2}{*}{ Mean } & \multirow[t]{2}{*}{$\mathrm{SD}$} & \multicolumn{2}{|c|}{ One-way ANOVA } & \multirow[t]{2}{*}{$R^{2 \mathrm{c}}$} & \multicolumn{3}{|c|}{ Genetic effect (B against A, single allele) } \\
\hline & & & & & F-value & $P^{\mathrm{b}}$ & & $\begin{array}{l}\text { Additive } \\
\text { effect (a) }\end{array}$ & $\begin{array}{l}\text { Dominance } \\
\text { effect (d) }\end{array}$ & $\mathrm{d} / \mathrm{a}^{\mathrm{d}}$ \\
\hline \multirow[t]{6}{*}{ BRS $184 \times$ WC2 } & \multirow[t]{3}{*}{$\mathrm{NoU}$} & $\begin{array}{l}\text { Sct_187: A } \\
\text { Sct_187: H }\end{array}$ & $\begin{array}{l}1.86 \\
0.14\end{array}$ & $\begin{array}{l}0.22 \\
0.41\end{array}$ & \multirow[t]{3}{*}{476.134} & \multirow[t]{3}{*}{ 4.3E-68 } & \multirow[t]{3}{*}{0.60} & \multirow[t]{3}{*}{-0.93} & \multirow[t]{3}{*}{-0.80} & \multirow[t]{3}{*}{0.86} \\
\hline & & Sct_187: B & 0.01 & 0.07 & & & & & & \\
\hline & & All & 0.46 & 0.79 & & & & & & \\
\hline & \multirow[t]{3}{*}{ SL } & $\begin{array}{l}\text { Sct_187: A } \\
\text { Sct_187: H }\end{array}$ & $\begin{array}{l}2.86 \\
0.20\end{array}$ & $\begin{array}{l}0.24 \\
0.63\end{array}$ & \multirow[t]{3}{*}{503.291} & \multirow[t]{3}{*}{$9.5 \mathrm{E}-70$} & \multirow[t]{3}{*}{0.60} & \multirow[t]{3}{*}{-1.42} & \multirow[t]{3}{*}{-1.24} & \multirow[t]{3}{*}{0.87} \\
\hline & & Sct_187: B & 0.02 & 0.12 & & & & & & \\
\hline & & All & 0.69 & 1.20 & & & & & & \\
\hline \multirow[t]{6}{*}{ BRS $184 \times$ WC5 1} & \multirow[t]{3}{*}{$\mathrm{NoU}$} & $\begin{array}{l}\text { Satt079: A } \\
\text { Satt079: H }\end{array}$ & $\begin{array}{l}2.19 \\
0.23\end{array}$ & $\begin{array}{l}0.21 \\
0.30\end{array}$ & \multirow[t]{3}{*}{1014.328} & \multirow[t]{3}{*}{$5.4 \mathrm{E}-78$} & \multirow[t]{3}{*}{0.73} & \multirow[t]{3}{*}{-1.09} & \multirow[t]{3}{*}{-0.87} & \multirow[t]{3}{*}{0.80} \\
\hline & & Satt079: B & 0.01 & 0.03 & & & & & & \\
\hline & & All & 0.67 & 0.94 & & & & & & \\
\hline & \multirow[t]{3}{*}{ SL } & $\begin{array}{l}\text { Satt079: A } \\
\text { Satt079: H }\end{array}$ & $\begin{array}{l}2.95 \\
0.31\end{array}$ & $\begin{array}{l}0.11 \\
0.37\end{array}$ & \multirow[t]{3}{*}{1439.283} & \multirow[t]{3}{*}{$5.1 \mathrm{E}-87$} & 0.74 & -1.47 & -1.17 & 0.80 \\
\hline & & Satt079: B & 0.01 & 0.03 & & & & & & \\
\hline & & All & 0.90 & 1.25 & & & & & & \\
\hline BRS $184 \times$ WC61 & $\mathrm{NoU}$ & $\begin{array}{l}\text { Satt079: A } \\
\text { Satt079: H }\end{array}$ & $\begin{array}{l}2.63 \\
1.12\end{array}$ & $\begin{array}{l}0.36 \\
0.63\end{array}$ & 134.201 & $3.1 \mathrm{E}-30$ & 0.68 & -1.18 & -0.34 & 0.29 \\
\hline & & Satt079: B & 0.28 & 0.39 & & & & & & \\
\hline & & All & 1.17 & 0.96 & & & & & & \\
\hline & SL & $\begin{array}{l}\text { Satt079: A } \\
\text { Satt079: H }\end{array}$ & $\begin{array}{l}3.00 \\
1.51\end{array}$ & $\begin{array}{l}0.02 \\
0.78\end{array}$ & 109.100 & 7.7E-27 & 0.66 & -1.31 & -0.17 & 0.13 \\
\hline & & Satt079: B & 0.38 & 0.59 & & & & & & \\
\hline & & All & 1.47 & 1.10 & & & & & & \\
\hline
\end{tabular}

$S D$, standard deviation; *NoU, number of uredinia per lesion; $S L$, sporulation level

${ }^{\text {a }}$ Marker genotype: A: homozygous susceptible (BRS 184), H: heterozygous, B: homozygous resistant (WC varieties)

${ }^{\mathrm{b}} P$ : probability of significance calculated by ANOVA

${ }^{\mathrm{c}} R^{2}$ : coefficient of determination calculated by regression analysis (for the selected marker)

${ }^{\mathrm{d}}$ degree of dominance: 1 complete dominance for resistance; 0 lack of dominance; -1 complete dominance for susceptibility

Publisher's note Springer Nature remains neutral with regard to jurisdictional claims in published maps and institutional affiliations. 